DOI: $10.17516 / 1999-494 X-0205$

УДК 62-52:656.56

\title{
Reliability Analysis in Planning of Traction Substation Reconstruction Based on Fuzzy Set Theory
}

\author{
Vladislav G. Belov* and Vladimir A. Tremyasov* \\ Siberian Federal University \\ Krasnoyarsk, Russian Federation
}

Received 18.12.2018, received in revised form 21.06.2019, accepted 21.01.2020

Abstract. The study proposes a probabilistic method using triangular fuzzy numbers to analyze the reliability of the traction substation. With this approach, the reliability assessment of the traction substation can be performed considering changes in the values of reliability indicators of electrical equipment, determined on the basis of the fuzzy set theory.

Keywords: fuzzy set theory, traction substation, reliability estimation, failure rate, uncertainty, minimum cuts, membership function.

Citation: Belov V.G., Tremyasov V.A. Reliability analysis in planning of traction substation reconstruction based on fuzzy set theory, J. Sib. Fed. Univ. Eng. \& Technol., 2020, 13(1), 52-62. DOI: 10.17516/1999-494X-0205

\section{Анализ надежности при планировании реконструкции \\ тяговой подстанции}

на основе теории нечетких множеств

\author{
В.Г. Белов, В.А. Тремясов \\ Сибирский федеральный университет \\ Российская Федерачия, Красноярск
}

Аннотащия. В работе для анализа надежности тяговой подстанции предлагается вероятностный метод с использованием треугольных нечетких чисел. С этим подходом оценка надежности схемы подстанции может быть выполнена с учетом изменений значения показателей надежности электрооборудования, определенных на основе теории нечетких множеств.

(C) Siberian Federal University. All rights reserved

This work is licensed under a Creative Commons Attribution-NonCommercial 4.0 International License (CC BY-NC 4.0).

* Corresponding author E-mail address: belov_v2012@mail.ru 
Ключевые слова: теория нечетких множеств, тяговая подстанция, оценка надежности, интенсивность отказов, неопределенность, минимальное сечение, функция принадлежности.

Цитирование: Белов, В.Г. Анализ надежности при планировании реконструкции тяговой подстанции на основе теории нечетких множеств / В.Г. Белов, В.А. Тремясов // Журн. Сиб. федер. ун-та. Техника и технологии, 2020. 13(1). C. 52-62. DOI: 10.17516/1999-494X-0205

\section{Введение}

Железнодорожный транспорт представляет собой сложную техническую систему. Одним из показателей эффективности функционирования такой системы является надежность.

Согласно «Стратегии развития железнодорожного транспорта в Российской Федерации до 2030 года» [1] предусматривается ввод новых и реконструкция существующих тяговых подстанций, строительство новых линий контактной сети. На этапе проектирования расчет показателей надежности тяговой подстанции осуществляется в основном классическими методами [2-4] на основе составления структурных схем и использования статистических значений показателей надежности отдельных элементов. Краеугольным камнем большинства методов оценок надежности служит еще и тот факт, что зачастую за исходные показатели надежности принимают значения показателей надежности нового оборудования, что не является обоснованным в процессе эксплуатации и планировании реконструкции тяговой подстанции.

В системах тягового электроснабжения (СТЭ) есть два типа неопределенности: случайность и нечеткость [5]. Вероятностные модели могут использоваться для случайности, но не для нечеткости. На практике при анализе надежности вариантов реконструкции тяговых подстанций из-за неточности и неполноты исходных данных оценка точных значений показателей надежности становится затруднительной. Лица, принимающие решение (ЛПР), в таких случаях рассматривают приближенные значения показателей, а доверительные границы таких показателей надежности могут отличаться от реальных значений. Принимая во внимание неопределенность и неполноту сведений об условиях эксплуатации конкретного электрооборудования, представляется обоснованным использовать модели надежности с нечеткими параметрами $[5,6]$. Нечеткие модели становятся необходимым дополнением к вероятностным моделям, чтобы учесть оба вида неопределенности исходных данных при вероятностном планировании реконструкции и модернизации тяговой подстанции.

Вышесказанное делает актуальным разработку и совершенствование методов анализа надежности тяговой подстанции при реконструкции в условиях неопределенности.

Один из методов, который позволяет вычислить показатели надежности в условиях неопределенности, основан на применении теории нечетких множеств (ТНM) [5, 7]. В работах [8-11] были разработаны методы с применением так называемых треугольных нечетких чисел (ТНЧ), представляющих собой нечеткие числа с функцией принадлежности (ФП) в треугольном виде. ТНМ - математический аппарат для работы с объектами, не имеющими жестких, однозначно задаваемых границ. Он позволяет формально описывать нестрогие, нечеткие, расплывчатые понятия и производить с ними различные операции.

На основе ТНЧ предлагается определить показатели надежности элементов тяговой подстанции, что позволит на основе этих показателей в сочетании с методом минимальных се- 
чений отказов [12] выполнить анализ надежности схемы электрических соединений тяговой подстанции в условиях неопределенности.

\section{Понятие нечеткого числа и правила операций}

Пусть $U$ - традиционное множество и его член обозначается как $x$. Нечеткое множество $A$ по $U$ определено как ряд упорядоченных пар и выражается в виде

$$
A=\left\{\left(x, \mu_{A}(x) \mid x \in U\right\},\right.
$$

где $\mu_{\mathrm{A}}(x)-$ ФП элемента к множеству $A$, изменяющаяся от 0 до 1.

Равенство $\mu_{\mathrm{A}}(x)=1$ означает, что $x$ точно принадлежит множеству $A$; равенство $\mu_{A}(x)=0$ говорит о том, что $x$ точно не принадлежит множеству $A$. Нечеткие множества отличаются от обычных множеств тем, что допускают промежуточные значения функции принадлежности, например, $\mu_{A}(x)=0,5$.

В частности, $A$ - вероятностное нечеткое множество, если $\mu_{A}(x)$ является случайной переменной, определенной на вероятностном пространстве.

$A_{\alpha}$, называемое $\alpha$ сечением $A$, определяется как

$$
A_{\alpha}=\left\{\left(x \in U \mid \mu_{A}(x) \geq a, 0<a \leq 1,\right\} .\right.
$$

Нечеткое число - специальный тип нечеткого множества. Нечеткое число определяется как выпуклое, нормализованное нечеткое множество с кусочно-непрерывной ФП [13]. Согласно этому определению очевидно, что $\alpha$ сечение $A_{\alpha}$ нечеткого числа $A$ является интервалом с нижней и верхней границей при $a_{l}(\alpha) \leq a_{u}(\alpha)$. Очевидно, что $a_{l}(\alpha)$ и $a_{u}(\alpha)$ - монотонно убывающая функция $\alpha$. И нижняя, и верхняя границы соответственно. Поэтому операции нечетких чисел могут быть выполнены по правилам вычисления интервалов.

Для двух заданных нечетких чисел $A_{\alpha}=\left[a_{l}(\alpha), a_{u}(\alpha)\right]$ и $B_{\alpha}=\left[b_{l}(\alpha), b_{u}(\alpha)\right]$ могут применяться следующие правила операций.

Суммирование

$$
(A+B)_{\alpha}=\left[a_{l}(\alpha)+b_{l}(\alpha), a_{u}(\alpha)+b_{u}(\alpha)\right]
$$

Вычитание

$$
(A-B)_{\alpha}=\left[a_{l}(\alpha)-b_{l}(\alpha), a_{u}(\alpha)-b_{u}(\alpha)\right]
$$

Умножение

$$
(A B)_{\alpha}=\left[\begin{array}{l}
\min \left(a_{l}(\alpha) \cdot b_{l}(\alpha), a_{u}(\alpha) \cdot b_{l}(\alpha), a_{l}(\alpha) \cdot b_{u}(\alpha), a_{u}(\alpha) \cdot b_{u}(\alpha)\right), \\
\max \left(a_{l}(\alpha) \cdot b_{l}(\alpha), a_{u}(\alpha) \cdot b_{l}(\alpha), a_{l}(\alpha) \cdot b_{u}(\alpha), a_{u}(\alpha) \cdot b_{u}(\alpha)\right)
\end{array}\right] .
$$

Если $A$ и $B$ определены на положительном монотонном пространстве вещественных чисел, то (5) становится

$$
(A B)_{\alpha}=\left[a_{l}(\alpha) \cdot b_{l}(\alpha), a_{u}(\alpha) \cdot b_{u}(\alpha)\right] .
$$

В частности, если $H$ - положительное постоянное число, то 


$$
(H A)_{\alpha}=\left[H a_{l}(\alpha), H a_{u}(\alpha)\right]
$$

Деление

$$
\left(\frac{A}{B}\right)_{\alpha}=\left[a_{l}(\alpha) \cdot a_{u}(\alpha)\right] \cdot\left[\frac{1}{b_{l}(\alpha)}, \frac{1}{b_{u}(\alpha)}\right],
$$

где $b_{l}(\alpha) \neq 0$ и $b_{u}(\alpha) \neq 0$. Иначе, один или оба конца интервала расширяются до $\infty$.

Для практических вычислений удобно работать с нечеткими числами специального вида $[5,10]$.

Треугольное нечеткое число, которое часто обозначается как $A=\left(a_{1}, a_{2}, a_{3}\right)$, определено следующей ФП (рис. 1):

$$
\mu_{A}(x)= \begin{cases}\left(x-a_{1}\right) /\left(a_{2}-a_{1}\right) & \text { если } a_{1} \leq x \leq a_{2} \\ \left(a_{3}-x\right) /\left(a_{3}-a_{2}\right) & \text { если } a_{2} \leq x \leq a_{3} \\ 0 & \text { если } x \leq a_{1} \text { или } x \geq a_{3} .\end{cases}
$$

Вычисление $\alpha$ сечения $A_{\alpha}$ треугольного нечеткого числа

$$
A_{\alpha}=\left[a_{1}+\alpha\left(a_{2}-a_{1}\right), a_{3}-\alpha\left(a_{3}-a_{2}\right)\right] .
$$

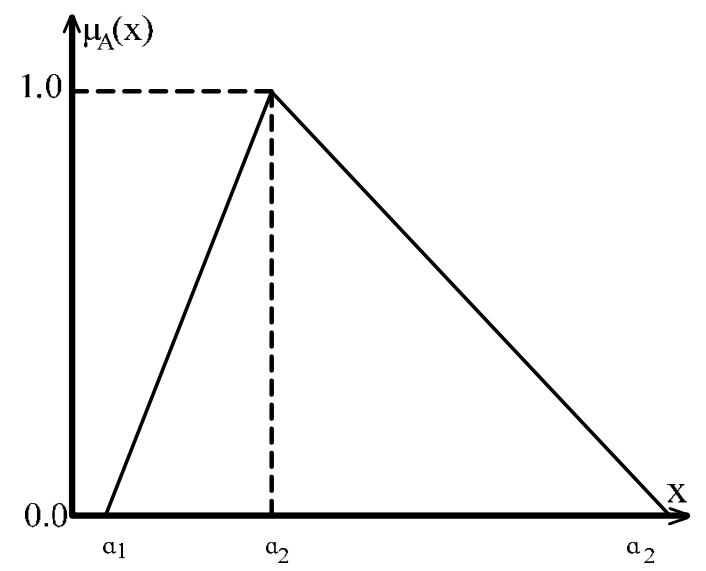

Рис. 1. ФП треугольного нечеткого числа

Fig. 1. Membership functions of a triangular fuzzy number

\section{Определение нечетких показателей надежности}

Элементы СТЭ классифицируются на две категории: с восстановлением и без восстановления. Элемент системы электроснабжения является восстанавливаемым в течение нормального периода эксплуатации и может выйти из строя в конце срока эксплуатации. Полнота исходной информации зависит от нескольких факторов [13]. По ряду показателей актуальная информация может отсутствовать $[6,14,15]$.

Среднее выборки времени восстановления может быть легко вычислено как среднеарифметическое времени восстановления при различных событиях отключений 


$$
\bar{\tau}=\frac{1}{n} \sum_{t=1}^{n} \tau_{i},
$$

где $\bar{\tau}$ - точечная оценка времени восстановления (в часах); $\tau_{i}$-время $i$-го восстановления; $n-$ число восстановлений в статистических отчетах.

Доверительный интервал ожидаемого времени восстановления может быть оценен по критериям $t$-распределения или нормального распределения $[5,10]$.

Метод оценки следующий. Предполагается, что $\beta$ представляет реально ожидаемое время восстановления, а $s$ - среднеквадратичное времени восстановления по выборке. Если используется критерий $t$-распределения, то можно подтвердить, что для данного существования уровня $\alpha$ случайная переменная $(\bar{\tau}-\beta) \sqrt{n} / s$ расположена между $-t_{\alpha}(n-1)$ и $t_{\alpha}(n-1)$ с вероятностью $1-\alpha$, где $t_{\alpha}(n-1)$ - такое значение, что интеграл функции плотности $t$-распределения с $(n-1)$ степенями свободы от $t_{\alpha}(n-1)$ до $\infty$ равняется $\alpha$. Поэтому мы имеем

$$
-t_{\alpha}(n-1) \leq \frac{(\bar{\tau}-\beta)}{s / \sqrt{n}} \leq t_{\alpha}(n-1) .
$$

Средняя интенсивность отказов отдельных компонентов не может быть получена как среднее выборки из отчетов по отказам. Интенсивность отказов оценивается как среднее число отказов за год в течение рассматриваемого периода времени $T$ :

$$
\bar{\lambda}=\frac{n}{T}
$$

где $\bar{\lambda}$ - точечная оценка интенсивности отказов (отказов/год); $n$ - число отказов элемента за рассматриваемое время $T$ (в годах), которое представляет все прошедшее время минус полное время отключений из-за отказов. В большинстве случаев полное время отключений - очень малая часть и $T$ может быть аппроксимировано всем прошедшим временем, в течение которого рассматриваются события отказов. Это подразумевает, что интенсивность отказов аппроксимирована частотой отказов.

Доверительный интервал ожидаемой интенсивности отказов может быть оценен следующим методом. Согласно теории статистики существуют следующие соотношения между распределением $\chi^{2}$ (хи-квадрат) и распределением Пуассона:

$$
\chi^{2}(2 N)=2 \lambda T
$$

Здесь $\lambda$ - ожидаемая интенсивность отказов, $T$ - весь рассматриваемый период времени, $N$ - число отказов за время $T$.

Уравнение (14) указывает, что двукратная величина отказов в течение времени $T$ следует распределению хи-квадрат с $2 N$ степенями свободы. Поэтому для заданного уровня значения $\alpha$ можно утверждать, что интенсивность отказов $\lambda$ относится к следующему случайному доверительному интервалу с вероятностью 1- $\alpha$ :

$$
\lambda^{\prime}=\frac{\chi_{1-\alpha}^{2}(2 N)}{2 T} \leq \lambda \leq \frac{\chi_{\alpha}^{2}(2 N)}{2 T}=\lambda^{\prime \prime} .
$$


Вычисления нечетких чисел, по существу, связаны с обнаружением точных верхней и нижней границ нечеткой функции [10]. При необходимости с помощью экспертов может быть получена более консервативная (более широкая) интервальная оценка [8].

Коэффициент простоя для восстанавливаемого $j$-го элемента равен

$$
q_{j}(t)_{\alpha}=\frac{\left(\lambda_{j}\right)_{\alpha}}{\left(\left(\lambda_{j}\right)_{\alpha}+\left(\frac{1}{\tau_{j}}\right)_{\alpha}\right)} \approx \frac{\left(\lambda_{j}\right)_{\alpha}}{\left(\frac{1}{\tau_{j}}\right)_{\alpha}}=\left(\lambda_{j}\right)_{\alpha} \cdot\left(\tau_{j}\right)_{\alpha}=\left[\lambda_{j}{ }^{\prime} \cdot \tau_{j}{ }^{\prime} ; \overline{\lambda_{j}} \cdot \overline{\tau_{j}} ; \lambda_{j}{ }^{\prime \prime} \cdot \tau_{j}{ }_{j}\right],
$$

где $\left(\lambda_{j}\right)_{\alpha}$ - нечеткое число интенсивности отказов, записанное в виде $\left(\lambda_{j}\right)_{\alpha}=\left(\lambda_{j}, \overline{\lambda_{j}}, \lambda_{j}\right.$ ”), с нижней границей $\lambda_{j}$, средним значением $\overline{\lambda_{j}}$ и верхней границей $\lambda_{j}$ ”; $\left(\tau_{j}\right)_{\alpha}-$ нечеткое число времени восстановления, записанное в виде $\left(\tau_{j}\right)_{\alpha}=\left(\tau_{j}, \overline{\tau_{j}}, \tau_{j}{ }^{\prime \prime}\right)$, с нижней границей $\tau_{j}$, средним значением $\overline{\tau_{j}}$ и верхней границей $\tau_{j}{ }^{\prime}$.

Минимальное сечение отказов (МСО) определяется как набор элементов, отказ которых приводит к отказу системы, но если любой элемент сечения остается работоспособен, отказа системы нет.

В большинстве методов с использованием МСО часто принимают два вида приближений:

1) нет необходимости перечислять все $\mathrm{MCO}$, так как вероятности отказов элементов в целом малы и, таким образом, вероятность появления сечений более высокого порядка может быть очень низкой, т.е. МСО более высокого порядка могут быть проигнорированы в перечислении;

2) вероятности пересечений двух и более МСО во многих случаях обычно чрезвычайно низки, и поэтому влияния невзаимного исключения среди МСО незначительны.

Каждое МСО составлено из параллельных элементов, если для отказа набора должны отказать все элементы в наборе. Совокупность МСО соединена последовательно, если для отказа системы достаточно отказа только одного из них. Поэтому для модели системы может использоваться комбинация МСО последовательно и элементов каждого сечения параллельно.

ФП множества показателей для каждого МСО вычисляют, используя формулы надежности для параллельной и последовательной сети и правила операций для нечетких чисел [10].

МСО в расчетной схеме реализуется, если все исходные события (отказы элементов) $A_{1} \ldots$ $A_{n}$ происходят в нем. Вероятность возникновения $i$-го МСО в момент времени $t,\left(q_{i}^{*}(t)\right)_{\alpha}$ получаем при пересечении (конъюнкции) отказов элементов [12]:

$$
\left(q_{i}^{*}(t)\right)_{\alpha}=P\left(\left(A_{i}\right)_{\alpha} \cdot\left(A_{j}\right)_{\alpha}\right),
$$

где $\left(A_{i}\right)_{\alpha}-i$-е минимальное сечение, выраженное нечетким числом.

Показатель $\omega_{i}{ }^{*}(t)$ - ожидаемое число появлений $i$-го МСО в единицу времени в момент $t-$ определяется выражением

$$
\left(\omega_{i}^{*}(t)\right)_{\alpha}=\sum_{j=1}^{n}\left(\omega_{j}^{\prime} ; \overline{\omega_{j}} ; \omega_{j}^{\prime \prime}\right) \prod_{\frac{l=1}{l \neq j}}^{n}\left(q_{i}^{\prime} ; \overline{q_{i}} ; q_{i}^{\prime \prime}\right),
$$

где $\left(\omega_{j}(t)\right)_{\alpha}-$ нечеткое число, параметр потока $j$-го исходного события в $i$-м $\mathrm{MCO}, n$ - число членов МСО. 
Показатель $\left(\lambda_{\mathrm{i}}{ }^{*}(t)\right)_{\alpha}-$ интенсивность появления $i$-го $\mathrm{MCO}$ - определяется через показатели $\left(\omega_{i}^{*}(t)\right)_{\alpha}$ и $\left(q_{i}(t)\right)_{\alpha}:$

$$
\left(\lambda_{i}^{*}(t)\right)_{\alpha}=\frac{\left(\omega_{i}^{*}(t)\right)_{\alpha}}{\left[1-\left(q_{i}^{*}(t)\right)_{\alpha}\right]}=\frac{\sum_{j=1}^{n}\left(\omega_{j}{ }^{\prime} ; \overline{\omega_{j}} ; \omega_{j}{ }^{\prime \prime}\right) \prod_{\frac{l=1}{l \neq j}}^{n}\left(q_{i}{ }^{\prime} ; \overline{q_{i}} ; q_{i}{ }^{\prime \prime}\right)}{\left[1-P\left(\left(A_{i}\right)_{\alpha} \cdot\left(A_{j}\right)_{\alpha}\right)\right]} .
$$

Коэффициент простоя тяговой подстанции $\left(q_{c}(t)\right)_{\alpha}$ - вероятность того, что конечное событие отказа подстанции существует в момент $t$, т.е. вероятность отказа тяговой подстанции определяется по выражению

$$
\left.\left(q_{c}(t)\right)_{\alpha}=\sum_{i=1}^{N_{M C O}}\left(q_{i}^{*}(t)\right)_{\alpha}\right),
$$

где $N_{\text {мсо }}$ общее число МСО.

Интенсивность отказа тяговой подстанции $\left(\Lambda_{c}(t)\right)_{\alpha}$, или вероятность того, что конечное событие произойдет в единицу времени в момент времени $t$ при условии, что оно не существует в момент $t$,

$$
\left(\Lambda_{c}(t)\right)_{\alpha}=\sum_{i=1}^{N_{c}}\left(\lambda_{i}^{*}(t)\right)_{\alpha}
$$

ФП интенсивности отказов и времени восстановления элементов могут быть созданы по точечным и интервальным оценкам интенсивности отказов и времени восстановления соответственно. ФП коэффициента простоя элемента может быть получена по функциям принадлежности $\lambda$ и $\tau$.

Пример. Рассмотрим схему открытого распределительного устройства 110 кВ тяговой подстанции, показанную на рис. 2. В процессе реконструкции подстанции предлагается взамен устаревших масляных выключателей установить современные и более надежные элегазовые

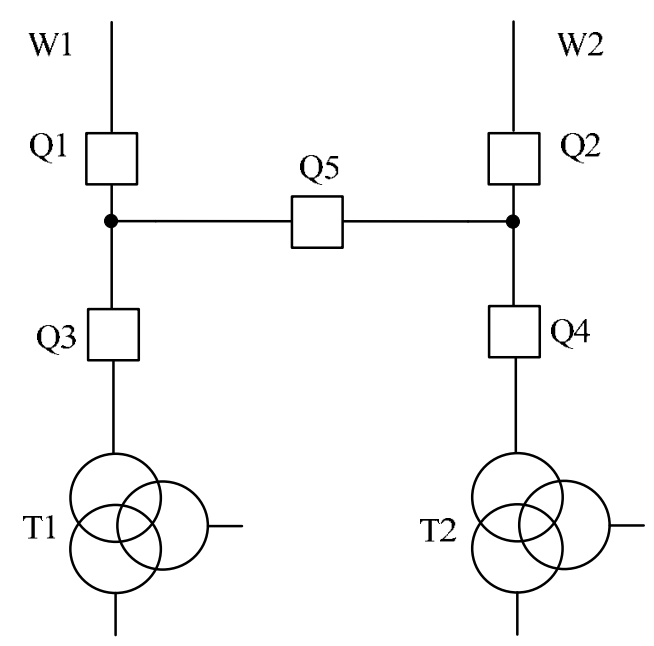

Рис. 2. Схема ОРУ 110 кВ тяговой подстанции

Fig. 2. $110 \mathrm{kV}$ open switchgear circuit of a traction substation 
аппараты. Средние значения и доверительные границы интенсивности отказов и времени восстановления электрооборудования тяговой подстанции представлены в табл. 1.

Значения нижней и верхней границ коэффициента простоя и интенсивности отказов тяговой подстанции, соответствующие пяти точкам функции принадлежности, даны в табл. 2 и 3 соответственно. В результатах использовали шаг 0,25 . Шаг может быть выбран в зависимости от требований точности при вычислении ФП.

Для двух вариантов схем тяговой подстанции с масляными и элегазовыми выключателями функции принадлежности показаны на рис. 3 и 4.

Анализ функций принадлежности показателей надежности (рис. 3, 4) показал, что применение элегазовых выключателей при реконструкции схемы тяговой подстанции приводит к повышению надежности ее в части уменьшения коэффициента простоя и интенсивности отказов.

Таблица 1. Средние значения и доверительные границы интенсивности отказов и времени восстановления электрооборудования тяговой подстанции

Table 1. Mean values and confidence limits of failure rate and recovery time of electrical equipment of traction substation

\begin{tabular}{|c|c|c|c|c|}
\hline \multicolumn{5}{|c|}{ Интенсивность отказов } \\
\hline Элемент схемы & Буквенное обозначение & $\lambda^{H}, 1 /$ год & $\lambda^{B}, 1 /$ год & $\bar{\lambda}, 1 /$ год \\
\hline Линия (5 км) & $\mathrm{W} 1, \mathrm{~W} 2$ & 0,072 & 0,1 & 0,08 \\
\hline Трансформатор & $\mathrm{T} 1, \mathrm{~T} 2$ & 0,012 & 0,02 & 0,014 \\
\hline Масляный выключатель & $\mathrm{Q} 1, \mathrm{Q} 2, \mathrm{Q} 3, \mathrm{Q} 4, \mathrm{Q} 5$ & 0,014 & 0,02 & 0,016 \\
\hline Элегазовый выключатель & $\mathrm{Q} 1, \mathrm{Q} 2, \mathrm{Q} 3, \mathrm{Q} 4, \mathrm{Q} 5$ & 0,0045 & 0,00625 & 0,005 \\
\hline \multicolumn{5}{|c|}{ Время восстановления } \\
\hline Элемент схемы & Буквенное обозначение & $\tau^{H}$, г & $\tau^{B}$, г & $\bar{\tau}$, г \\
\hline Линия (5 км) & $\mathrm{W} 1, \mathrm{~W} 2$ & 0,00153 & 0,00212 & 0,0017 \\
\hline Трансформатор & $\mathrm{T} 1, \mathrm{~T} 2$ & 0,00679 & 0,011 & 0,00799 \\
\hline Масляный выключатель & $\mathrm{Q} 1, \mathrm{Q} 2, \mathrm{Q} 3, \mathrm{Q} 4, \mathrm{Q} 5$ & 0,00414 & 0,00575 & 0,0046 \\
\hline Элегазовый выключатель & $\mathrm{Q} 1, \mathrm{Q} 2, \mathrm{Q} 3, \mathrm{Q} 4, \mathrm{Q} 5$ & 0,00205 & 0,00285 & 0,00228 \\
\hline
\end{tabular}

Таблица 2. ФП коэффициента простоя системы

Table 2. The membership functions of the ratio of system downtime

\begin{tabular}{|c|c|c|c|c|}
\hline \multirow{2}{*}{$\begin{array}{c}\text { Точка } \\
\text { принадлежности }\end{array}$} & \multicolumn{2}{|c|}{ ТП с масляными выключателями } & \multicolumn{2}{|c|}{ ТП с элегазовыми выключателями } \\
\cline { 2 - 5 } & Нижняя граница & Верхняя граница & Нижняя граница & Верхняя граница \\
\hline 1 & $7,89 \cdot 10^{-8}$ & $7,89 \cdot 10^{-8}$ & $3,78 \cdot 10^{-8}$ & $3,78 \cdot 10^{-8}$ \\
\hline 0,75 & $7,15 \cdot 10^{-8}$ & $1,14 \cdot 10^{-7}$ & $3,40 \cdot 10^{-8}$ & $5,64 \cdot 10^{-8}$ \\
\hline 0,5 & $6,35 \cdot 10^{-8}$ & $1,48 \cdot 10^{-7}$ & $3,02 \cdot 10^{-8}$ & $7,51 \cdot 10^{-8}$ \\
\hline 0,25 & $5,60 \cdot 10^{-8}$ & $1,84 \cdot 10^{-7}$ & $2,64 \cdot 10^{-8}$ & $9,39 \cdot 10^{-8}$ \\
\hline 0 & $4,76 \cdot 10^{-8}$ & $1,05 \cdot 10^{-7}$ & $2,26 \cdot 10^{-8}$ & $1,13 \cdot 10^{-7}$ \\
\hline
\end{tabular}


Таблица 3. ФП интенсивности отказов системы

Table 3. The membership function of the failure rate of the system

\begin{tabular}{|c|c|c|c|c|}
\hline \multirow{2}{*}{$\begin{array}{c}\text { Точка } \\
\text { принадлежности }\end{array}$} & \multicolumn{2}{|c|}{ ТП с масляными выключателями } & \multicolumn{2}{c|}{ ТП с элегазовыми выключателями } \\
\cline { 1 - 5 } & Нижняя граница & Верхняя граница & Нижняя граница & Верхняя граница \\
\hline 1 & $5,150 \cdot 10^{-5}$ & $5,150 \cdot 10^{-5}$ & $3,060 \cdot 10^{-5}$ & $3,060 \cdot 10^{-5}$ \\
\hline 0,75 & $4,770 \cdot 10^{-5}$ & $6,480 \cdot 10^{-5}$ & $2,810 \cdot 10^{-5}$ & $3,820 \cdot 10^{-5}$ \\
\hline 0,5 & $4,400 \cdot 10^{-5}$ & $7,820 \cdot 10^{-5}$ & $2,593 \cdot 10^{-5}$ & $4,618 \cdot 10^{-5}$ \\
\hline 0,25 & $4,033 \cdot 10^{-5}$ & $9,155 \cdot 10^{-5}$ & $2,376 \cdot 10^{-5}$ & $5,433 \cdot 10^{-5}$ \\
\hline 0 & $3,668 \cdot 10^{-5}$ & $1,053 \cdot 10^{-4}$ & $2,148 \cdot 10^{-5}$ & $6,233 \cdot 10^{-5}$ \\
\hline
\end{tabular}

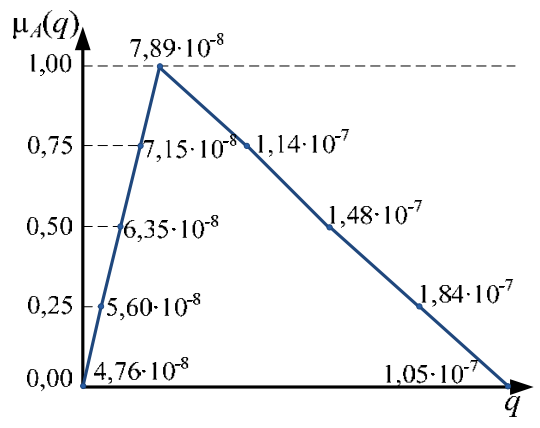

a)

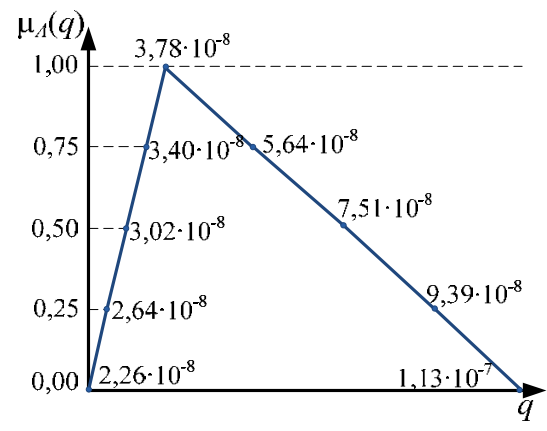

б)

Рис. 3. ФП коэффициентов простоя для тяговой подстанции с масляными (а) и элегазовыми (б) выключателями

Fig. 3. The function of the ratio of downtime for traction substation with a) oilbreakers switchgear and б) SF6 breakers switchgear

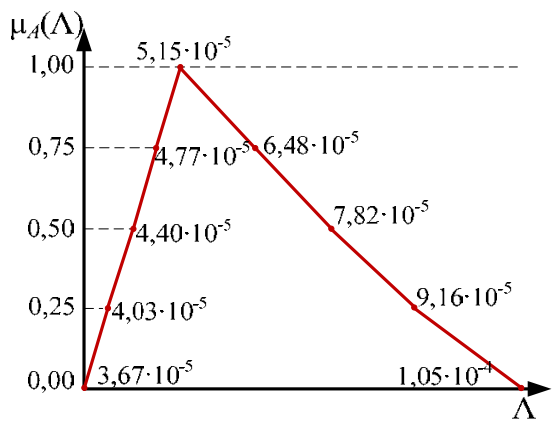

a)

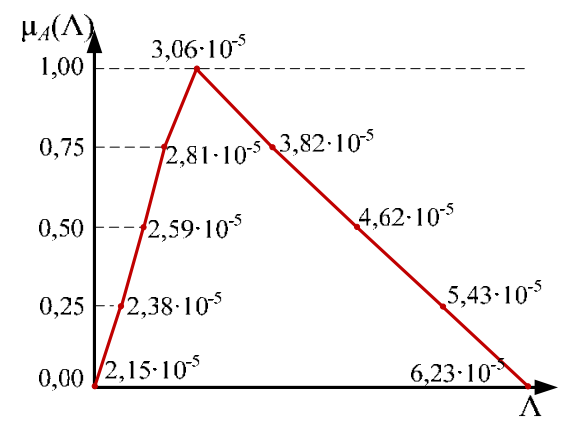

б)

Рис. 4. ФП интенсивности отказов для тяговой подстанции с масляными (а) и элегазовыми (б) выключателями

Fig. 4. The membership function of the failure rate for traction substations from a) oil breakers switchgear and б) SF6 breakers switchgear 


\section{Заключение}

Предложена методика анализа надежности при реконструкции тяговой подстанции на основе теории нечетких множеств, позволяющая оценить показатели надежности в условиях нечеткой информации исходных данных и принять обоснованный вариант реконструкции. Такой подход является актуальным при реконструкции тяговых подстанций железных дорог, когда объема исходных данных, используемых для оценки надежности, недостаточно. Показатели надежности элементов СТЭ могут быть описаны треугольными нечеткими числами.

Используемый метод построения функций принадлежности повышает эффективность решения задач оценки надежности тяговой подстанции для разного состава электрооборудования при выборе варианта реконструкции тяговой подстанции.

\section{Список литературы / References}

[1] Распоряжение Правительства РФ от 17.06.2008 N 877-р О Стратегии развития железнодорожного транспорта в Российской Федерации до 2030 года [Order of the Government of the Russian Federation of June 17, 2008 N 877-r On the Strategy for the Development of Railway Transport in the Russian Federation until 2030 (in Russian)]

[2] Дмитриев Е.И. Расчет надежности объектов инфраструктуры ОАО «РЖД». Актуальные проблемы авиации и космонавтики. Информационные технологии, 2011, 315 - 316 [Dmitriev E.I. Calculation of the reliability of infrastructure facilities of Russian Railways. Actual problems of aviation and astronautics. Information Technology, 2011, 315 - 316 (in Russian)]

[3] Гук Ю.Б. Теория и расчет надежности систем электроснабжения. Москва: Энергия, 1970, 177 c. [Guk Yu.B. Theory and calculation of reliability of power supply systems. Moscow, Energia, 1970, 177 p. (in Russian)]

[4] Воропай Н.И., Федотова Г.А. Направления и результаты исследований надежности систем энергетики. Надежность и безопасность энергетики. 2018, 11(4), 280-287 [Voropai N.I., Fedotova G.A. Areas and results of research on reliability of energy systems. Safety and Reliability of Power Industry, 2018, 11(4), 280-287 (in Russian)]

[5] Bai X. Fuzzy-based approaches to substation reliability evaluation. Electric Power Systems Research, May 2004, 69(2-3), 197-204.

[6] Zadeh L.A. Fuzzy Sets. Information Control, 1965, (8), 338-353.

[7] Орловский С.А. Проблемы принятия решений при нечеткой исходной информации. М.: Наука. Главная редакция физико-математической литературы, 1981, 208 c. [Orlovsky S.A. Decision making problems with fuzzy initial information. Moscow, Science. The main edition of the physical and mathematical literature, 1981, 208 p. (in Russian)]

[8] Bowles J.B., Pelaez C.E. Application of fuzzy logic to reliability engineering. Proceedings of the IEEE, March 1995, 83(3), 435-449.

[9] Zadeh L.A. Fuzzy sets as a basis for theory of possibility. Fuzzy Sets and Systems, 1978, (1), $3-28$.

[10] Li W., Xiong X., Zhou J. Fuzzy models of overhead power line weather-related outages. IEEE Trans. Power Sys. Aug. 2008, 23 (3), 1529-1531.

[11] Li W., Xiong X., Zhou J. Incorporating fuzzy models weather-related outages in transmission system relability assesment. IET Proceed. Generation, Transmiss. Distribut. Jan. 2009, 3(1), 26-37. 
[12] Тремясов В.А. Надежность электроснабжения. Красноярск: ИПЦ КГТУ, 2006, 163 с. [Tremyasov V.A. Reliability of power supply. Krasnoyarsk, CPI KSTU, 2006, 163 p. (in Russian)]

[13] Huang D., Nguang S.K. Robust control for uncertain networked control systems with random delays. Berlin, Springer-Verlag Berlin Heidelberg, 2009, 159 p.

[14] Саати Т. Принятие решений. Метод анализа иерархий. М.: Радио и связь, 1993, 278 с. [Saati T. Making decisions. Method of analysis of hierarchies. Moscow: Radio and communications, 1993, 78 p. (in Russian)]

[15] Пегат А. Нечеткое моделирование и управление. М.: Бином, 2009, 798 с. [Pegat A. Fuzzy modeling and control. Moscow, Binom, 2009, 798 p. (in Russian)] 